\title{
Article \\ Determination of cross-directional and cross-wall variations of passive biaxial mechanical properties of rat myocardium
}

\author{
Harry Ngwangwa ${ }^{1}$, Fulufhelo Nemavhola ${ }^{1 *}$, Thanyani Pandelani ${ }^{2}$, Makhosasana Msibi ${ }^{1}$, Israel Mabuda ${ }^{1}$, Neil Da- $^{2}$ \\ vies $^{3,4}$, Thomas Franz ${ }^{3,4,5,6,7}$
}

1 Unisa Biomedical Engineering Research Group, Department of Mechanical Engineering, School of Engineering, College of Science Engineering and Technology, University of South Africa, Pretoria, 0001, South Africa; masitfj@unisa.ac.za

2 Defence and security, Council for Scientific and Industrial Research (CSIR), Pretoria, 0001, South Africa; TPandelani@csir.co.za

3 Cardiovascular Research Unit, Department of Surgery, University of Cape Town, Observatory 7925, South Africa; Neil-Davies@uct.ac.za

4 Division of Biomedical Engineering, Department of Human Biology, University of Cape Town, Observatory 7925, South Africa; Thomas.Franz@uct.ac.za

5 Cardiovascular Research Unit, Department of Surgery, University of Cape Town, Observatory 7925, South Africa

6 Centre for High Performance Computing, Rosebank 7700, South Africa

7 Bioengineering Science Research Group, Engineering Sciences, Faculty of Engineering and the Environment, University of Southampton, Southampton SO171BJ, UK

* Correspondence: masitfj@unisa.ac.za ; Tel.: +27 114712354

\begin{abstract}
Heart myocardia are critical to the facilitation of heart pumping and blood circulating around the body. The biaxial mechanical testing of the Left Ventricle (RV) is utilised to build the computational model of the whole heart with little importance given to the unique mechanical properties of Right Ventricle (RV) and Mid-wall (MDW). Most of those studies focussed on the LV of the heart, and then apply the obtained characteristics with a few modifications to the right side of the heart. However, that view has been contested over time with the realisation that the right side of the heart possesses its own unique mechanical properties that are widely distinct from that of the left side of the heart. This paper is aimed at reporting and evaluating the passive mechanical property differences in the three main walls of the rat heart based on biaxial tensile test data. Fifteen mature Wistar rats weighing $225 \pm 25 \mathrm{~g}$ were euthanised by inhalation of $5 \%$ halothane. The hearts were excised after which all the top chambers comprising the two atria, pulmonary and vena cava trunks, aorta and valves are all dissected out. Then $5 \times 5 \mathrm{~mm}$ sections from the middle of each wall were carefully dissected with a surgical knife to avoid over-prestraining the specimens. The specimens were subjected tensile test. The elastic moduli, peak stresses in the toe region and stresses at $40 \%$ strain, anisotropy indices as well as the stored strain energy in the toe and linear region up to $40 \%$ strain are used for statistical significance tests. The following are the main findings of this study: (1) LV and MDW tissues have relatively shorter toe regions of $10-15 \%$ strain as compared to RV tissue whose toe region extends up to twice as much as that (2) LV tissues have higher strain energy storage in the linear region despite being lower in stiffness than the RV (3) the MDW has the highest strain energy storage along both directions which might be directly related to its high level of anisotropy. These findings, though for a specific animal species at similar age and around the same body mass, emphasise the importance of application of wall specific material parameters to obtain accurate ventricular hyperelastic models. The findings further enhance our understanding of the desired mechanical behaviour of the different ventricle walls.
\end{abstract}


Keywords: Myocardium tissue; Left ventricle mechanics; right ventricle mechanics; cardiac septum mechanics; anisotropy index

\section{Introduction}

Testing remains the most common modality of mechanical analysis for biological [16] and other soft materials [7]; however, biaxial testing enables a more comprehensive understanding of these materials' mechanical behaviour, which is also necessitated by the fact that heart muscle is spatially heterogenous. The physiological functioning of myocardial muscles involves a complex force pattern in which biaxial tensile and compressive forces during diastole and systole phases are combined with forces in the third dimension $[8,9]$. Therefore, it is important that the study of myocardial muscle behaviour be carried out along more than a single dimension. Some researchers $[8,10]$ recommended use of multiaxial analysis of cardiac tissue due to the intrinsic anisotropy and variation of myocyte fiber orientation and mechanical properties of myocardial tissues. It is stated that biaxial cardiac in vitro models can precisely represent the mechanobiological behaviour of cardiac tissue[11]. Besides, other studies [12] further recommended that for cardiac tissue constitutive modelling, more uniaxial or biaxial tensile tests, identification of viscoelastic properties in various time scales, use of pressure-volume curves be carried out, and that there is need for correlating the passive biomechanical cardiac tissue properties with microstructural observations.

Previous studies have been conducted to understand the tissue mechanics of healthy myocardia [13] to assist in the advancement of heart-specific computational models [1, 3, 14-19] and development of new materials for use in diagnosing and treatment of coronary illnesses. This study is aimed at investigating cross-direction and cross-wall variations in infarcted rat myocardium. Despite many studies which show that the myocardium tissue is complex, nonlinear, anisotropic, and heterogeneous, not enough study has been conducted on the nature of these complexities across the heart's three main walls, namely, the left ventricle (LV), cardiac septum (MDW) and the right ventricle (RV) [20]. In order to accurately bioengineer the heart muscle, it is essential that the passive mechanical behaviour of the real tissue of the entire heart be correctly understood and profiled. In addition, it is hard to accurately model the heart's mechanical response without sufficiently understanding its walls' mechanical properties, but those studies have been rare largely due to lack of sufficient research on the right ventricle and soft tissues on the right side of the heart[21-24]. The more muscular left ventricle has dominated most research on the heart due to its role in supplying blood to the entire body rather than that of the right ventricle which merely supplies blood to the lungs. In the following we highlight a few studies that have attempted to address different regions of the heart.

The mechanical properties data of the right ventricle free wall and its constitutive relationship were reported for the first time in the published literature in 1993 [25, 26]. They studied regional differences by excising tissue specimens from the conus and sinus regions of the mongrel dog's hearts. They found that the myocardium was more anisotropic in the conus direction with greater stiffness along the fiber direction. They further found that both anisotropy and fiber direction stiffness were greater in the RV than in LV. Much later, [27] found that different regions of the heart exhibited differences in material properties due to different sarcomere lengths. They also added that the RV is embryonically, structurally and functionally different from the LV, and further recommended that heart muscle research should, among other areas, focus on studies of tissues from different wall layers and ventricles in order to better understand its behaviour. Other workers [28] 
observed the differences in material behaviour among all six regions of the heart valves with the central region exhibiting more anisotropy and being more extensible toward the annulus than the edge regions. The authors further pointed out that it is difficult to model the heart valves correctly with models that assume material homogeneity over different regions when it is known that they are spatially heterogeneous. At a functionally macroscopic scale, some studies[24] have noted that the differences between the RV and LV myocardium are in terms of their stiffness in the fiber direction as well as the orientation and distribution of their fibers.

This paper is a sequel to an earlier study on investigation of regional dependencies on porcine myocardium [29] where it was reported that the material parameters obtained from fitting the Fung and Choi-Vito models to the myocardial biaxial tensile test data showed some regional dependencies. Therefore, in this study we focus on examining the nature of these differences by conducting single-factor analysis of variance on tissue elastic moduli (stiffness), precondition stress, and stress at $40 \%$ strain. These significance tests are specifically applied in cross-direction and cross-wall sense in order to detect both directional-dependent and regional-dependent variances.

\section{Materials and Methods}

\subsection{Tissue acquisition and preparation}

Fifteen $(\mathrm{N}=15)$ Wistar rats $(200-250 \mathrm{~g})$ aged between 8 and 10 weeks [30], were euthanised by inhalation of $5 \%$ halothane. This is in accordance with animal welfare regulations in South Africa concerning animal sacrifice to avoid mortis rigor [31, 32]. Once breathing and heartbeat had stopped, the heart was dissected out. The atria, pulmonary and vena cava trunks, the valves, and all soft tissues above the short axis of the heart were dissected away. The fresh rat hearts were then packaged in a temperature-controlled box maintained at between $0^{\circ} \mathrm{C}$ to $6^{\circ} \mathrm{C}$ from the University of Cape Town and delivered to the University of South Africa in less than 3 hours. The outflow direction was marked as the longitudinal direction $\left(0^{\circ}\right)$ and while that aligned with the heart wall's shorter diameter was denoted as the circumferential direction. Full cross-sections measuring approximately 5 x $5 \mathrm{~mm}$ were dissected from three different walls of the heart, namely, left ventricle (LV), cardiac septum (MDW), and right ventricle (RV). Sharp and sterilised surgical knives were used during the dissection to avoid overly pre-straining the specimens[33, 34]. The mechanical tests were then conducted immediately after receipt of the samples.

\subsection{Biaxial mechanical testing}

CellScale Biaxial testing system was used to measure the tensile response of all tissue samples. All prepared tissue samples were mounted in the custom biaxial tensile material testing apparatus (BioTester 5000 CellScalle, Wateroo, ON, Canada ${ }^{\circledR}$ ) specifically designed for soft tissue mechanical testing. The BioTester 5000 biaxial system is installed with a unique system that uses rakes for piercing the tissue. In this test, the four rakes (see Figure 1) are utilised to clamp the tissue sample for biaxial tensile testing. A total of 15 samples were harvested from the LV, MDW and RV and subjected to equi-biaxial tensile testing. The major dimensions such as length, width and the thickness of each sample were measured using a Vernier calliper. To ensure the accuracy of the measurement, each dimension was measured four times and average dimensions were then utilised for further processing. Before collecting data, the precondition was conducted by applying a 10 $\%$ strain on the sample at a strain rate of $0.001 / \mathrm{s}$. A preload of $5 \mathrm{mN}$ was applied for 0.53 seconds. To maintain hydration and mimicking the body temperature, saline $0.91 \% \mathrm{w} / \mathrm{v}$ of $\mathrm{NaCl}$ was placed in the bath and heated to $37{ }^{\circ} \mathrm{C}$ and maintained for the duration of testing. Each sample was subjected to $40 \%$ biaxial strain in the fibre and cross-fibre 
direction simultaneously at strain rate of $40 \%$ strain $/ 5$ seconds. $40 \%$ strain was selected to be the physiological magnitude of rat heart.

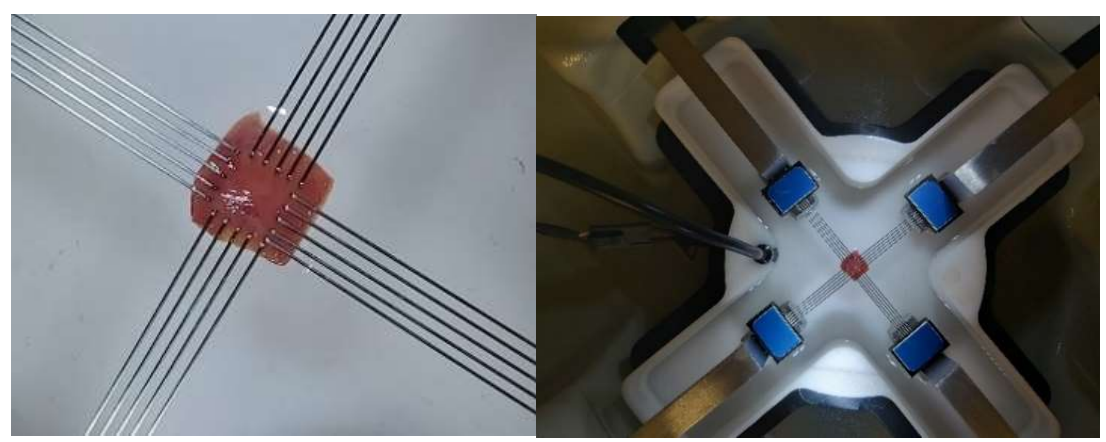

(a)

(b)

Figure 1. Experimental set-up of biaxial testing of rat heart passive myocardium. Figure 1 (a) shows the $5 \times 5 \mathrm{~mm}$ LV myocardium sample and rake assembly for clamping. Figure $1(b)$ shows the BioTester system used for biaxial testing of rat LV myocardium including the water bath for mimicking the body temperature of $37^{\circ} \mathrm{C}$.

\subsection{Tissue stress-strain analysis}

In this study the stresses were initially calculated using the first Piola-Kirchoff stress $T_{i j}$, then converted into the Cauchy stress $\sigma_{i j}$ via the relationship [26, 35]

$\sigma_{i j}=\lambda_{i} T_{i j}$

Where $\mathrm{i}$ and $\mathrm{j}$ denote tensorial indices (refer to figure 2) which for practical purposes in this case may be interpreted as the two different directions, $\lambda$ denotes the stretch ratio and

$T_{i j}=F_{i j} / A_{i j}$ where $\mathrm{F}$ is the normal force and $\mathrm{A}$ is the undeformed cross-sectional area. The infinitesimal strains in the two-directions were calculated by the formula:

$\varepsilon_{i j}=\frac{\partial u_{i j}}{\partial x_{i j}}$

Where $\mathrm{u}$ denotes the displacement tensor and $\mathrm{x}$ is the coordinate.

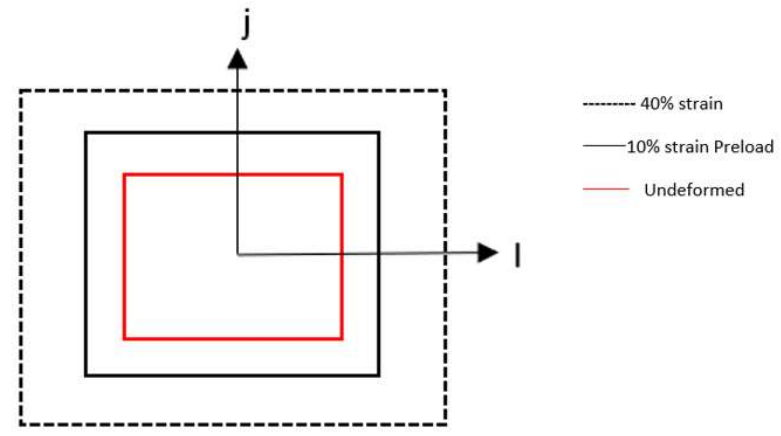

Figure 2. Stress/ strain direction undeformed and under different strain rate.

We assume that there are no shear stresses on the outer edges of the specimen therefore only normal stresses are considered in this paper. The calculated stress results are cut-off at $40 \%$ strain (refer to figure 2). These stress results however are noisy therefore they were further filtered with an 8-point moving average filter. The data were resampled and further smoothened using a quadratic filter.

\subsection{Analysis of variance}

The statistical analysis involved the evaluation of the significance tests using oneway single factor variance in Excel on the tissues based on the variations of elastic moduli, 
toe-region upper limit stress (preconditioning stress), and the stresses at $40 \%$ strain between the two directions for each wall, and their cross-wall variations along a particular direction. The rationale behind the first set of significance testing is to establish any statistical significance in the variances between tissue behaviour in the two directions for each myocardial tissue while the second set of significance test were aimed at investigating any differences from one wall to another for the same direction.

In the analysis of variance, the significance of the differences between any two given sets of data containing elastic modulus values is evaluated. The statistic called the P-value is used to indicate levels of significance in the variances. In this study, the null hypothesis is such that the variance in the given two sets of data is statistically significant if the Pvalue is less than or equal to 0.05 [36].

The elastic moduli were determined from the elastic region for each heart wall being between 13 and $37 \%$ strain for LV and MDW, and between 24 and $37 \%$ strain for RV, toe region peak stresses were picked at $12 \%$ strain for LV and MDW and $24 \%$ strain for RV. These investigations were conducted between the two different directions for all the three walls, and between one wall and another for each of the two directions.

\section{Experimental results}

\subsection{Stress-strain relationship}

The stress-strain curves in Figure 3-5 are overlayed with the standard error bounds to show how well the measured results lay within one-standard deviation bounds. Furthermore, average curves have been plotted over the same axes and for all the walls in both directions, and these curves lie mid-way. The stresses are plotted up to $40 \%$ strain in longitudinal and circumferential directions for all three walls of the heart. There is a total of fifteen tests for each wall (Test $I-X V)$. Though some of the stress-strain curves exhibit diminished toe regions probably due to differences in the preconditioning, the results show that the toe regions extend up to $12 \%$ strain for LV and MDW tissues and almost double as much for the RV. The plotted results show that the stress-strain curves divert away from the average curve upwards with increased strain within the linear region. Therefore, although almost all curves lie within the one-standard deviation margins, about $30 \%$ lie outside the those bounds in the upper ends of the linear region. Theoretically, it is expected that for normally distributed test data $68 \%$ should be within the onestandard deviation bounds which is in line with these test results [36]. This demonstrates the normality and quality of the test procedure in this study. There were not major differences in the test procedure from one test to another and any differences might have arisen from unavoidable disturbances such as clamping conditions, temperature fluctuations during testing, differences in test times from the time of animal sacrifice, and different pre-strain histories [34, 37]. 

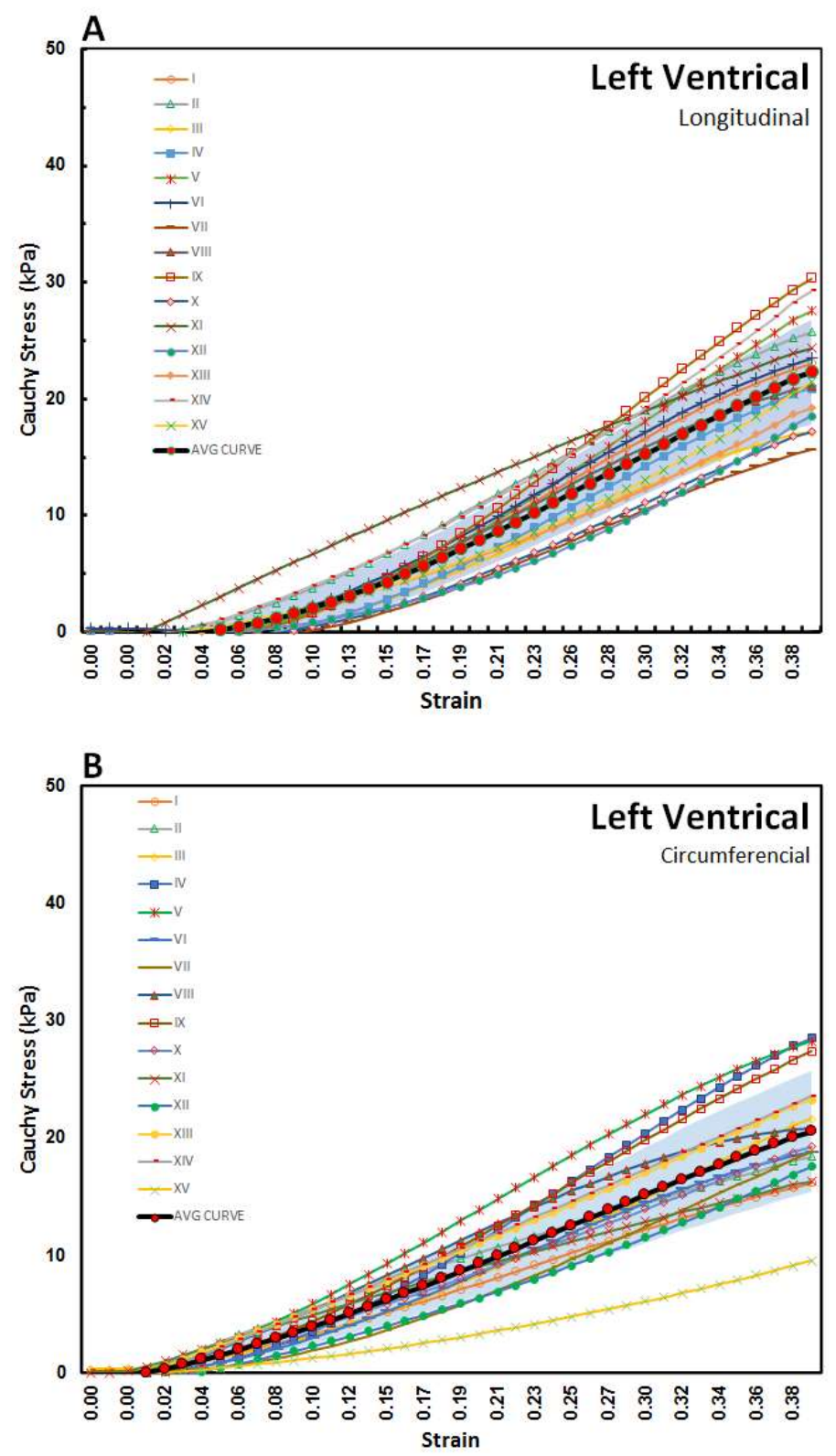

Figure 3. Stress-strain graphs for LV myocardium in the (a) longitudinal and (b) circumferential directions overlayed with standard error regions as well as an average curve. 

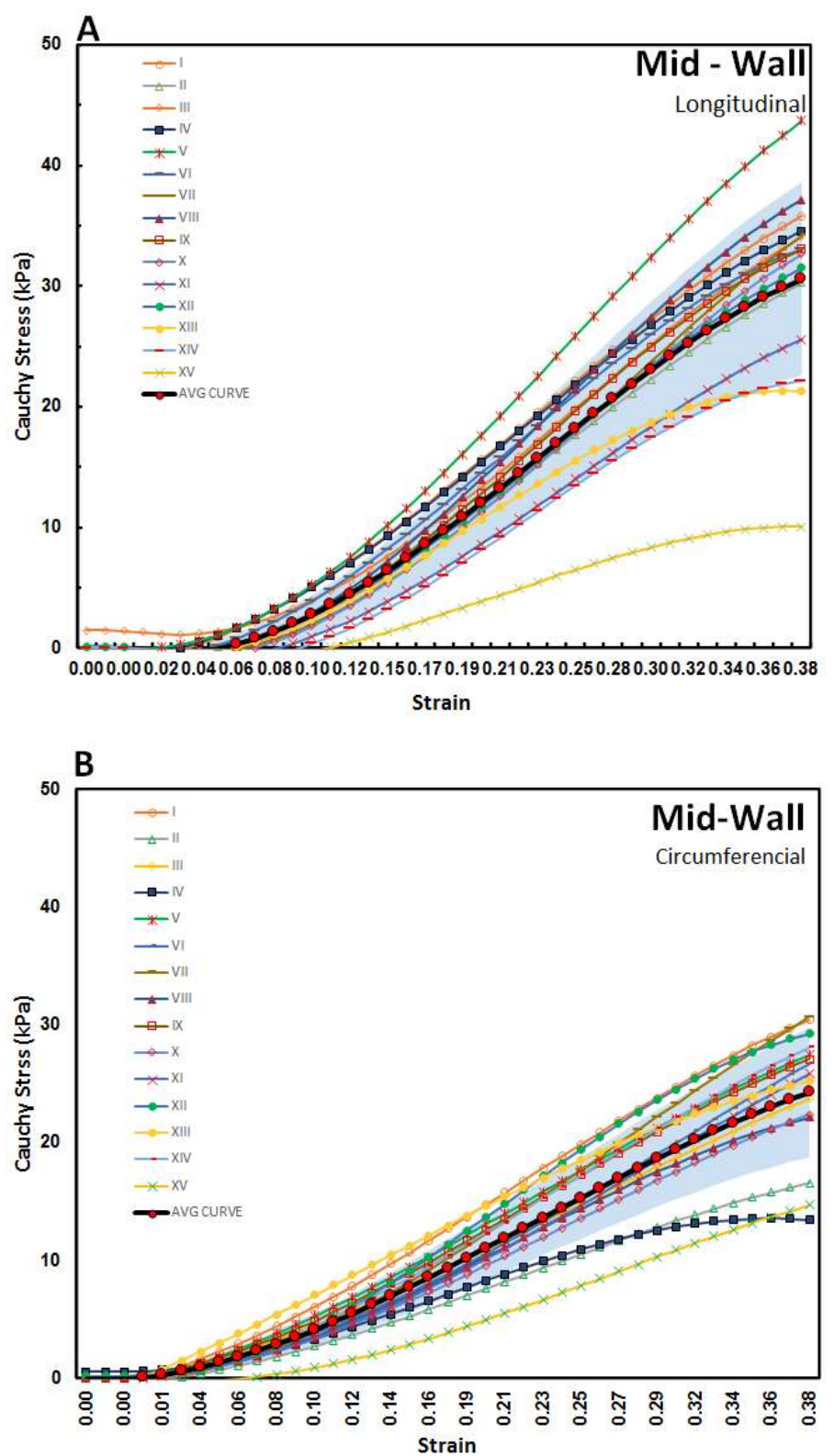

Figure 4. Stress-strain graphs for MDW myocardium in the (a) longitudinal and (b) circumferential directions overlayed with standard error regions as well as an average curve. 

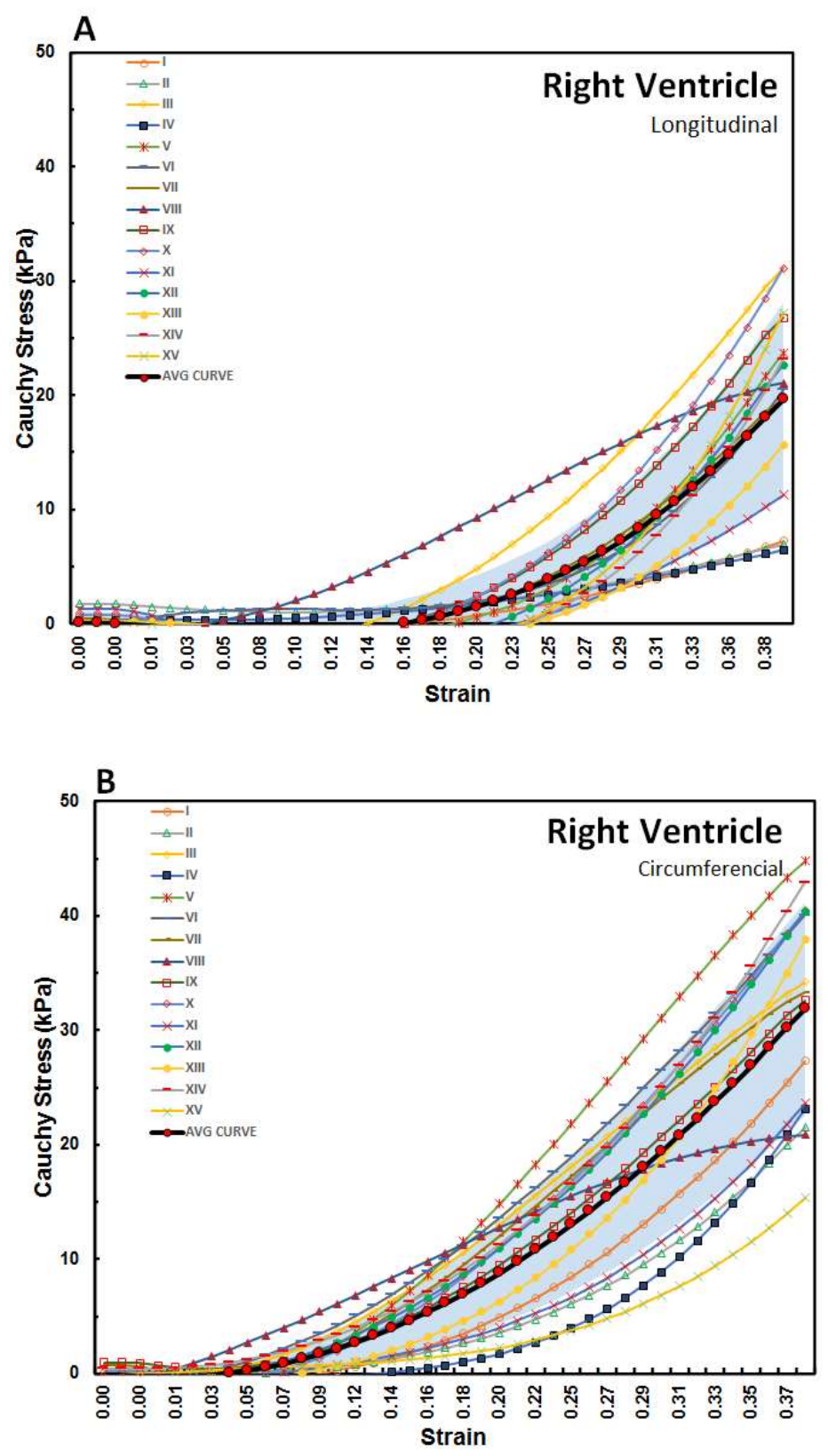

Figure 5. Stress-strain graphs for RV myocardium in the (a) longitudinal and (b) circumferential directions overlayed with standard error regions as well as an average curve.

To examine the exact trend of the stress-strain curves relative to the average curves, a standard error of the mean (SEM) was calculated and plotted in Figure 6. The SEM results also show that the errors from the average curve increase with increased strain in the linear region for both longitudinal and circumferential directions in all the three walls. Among the three walls, the largest SEM occurs in the RV wall. The circumferential direction consistently yields larger SEM than the longitudinal direction in all the three walls. 

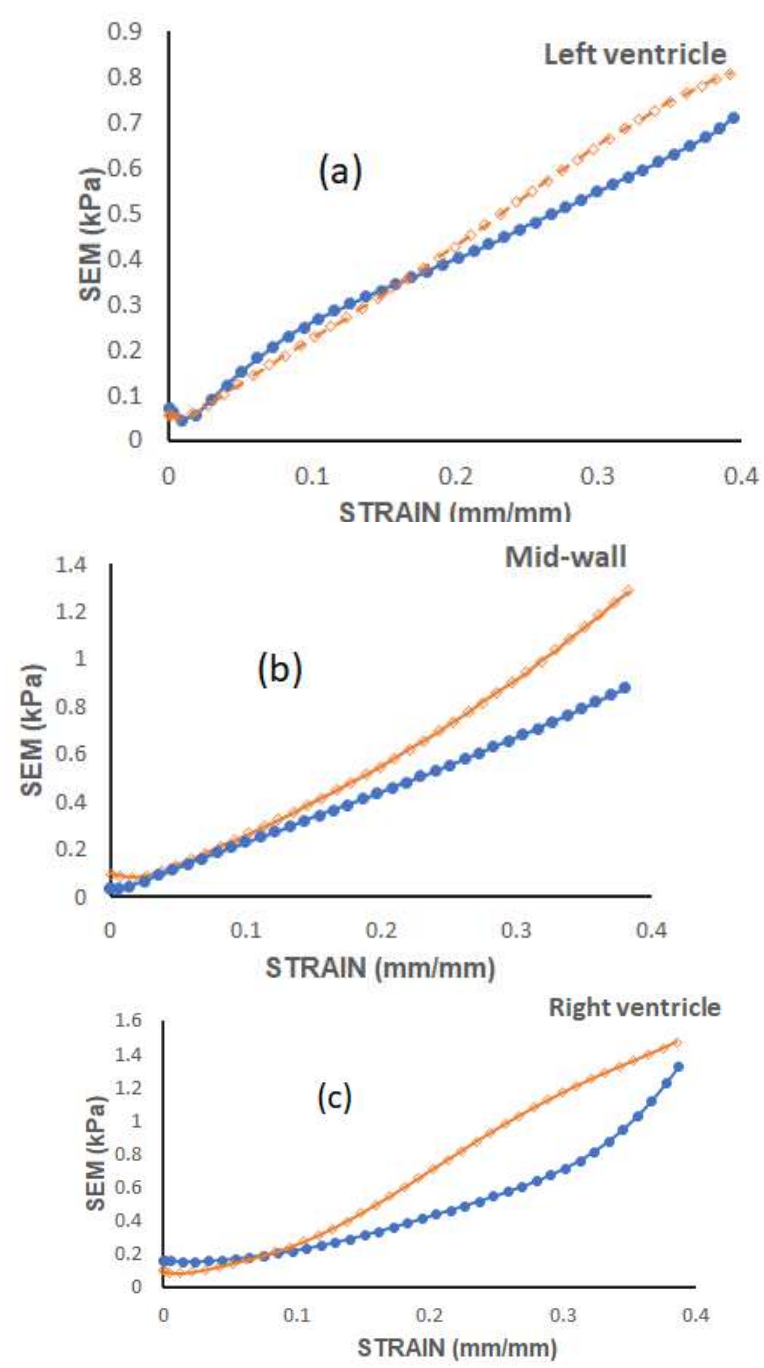

Figure 6. Standard error of the mean (SEM) for the three heart walls along each direction.

\subsection{Cross-directional variation}

Figure 7 shows the average stress-strain curves along the longitudinal and circumferential directions plotted on the same axes for each wall. For the LV and MDW the tissues exhibit qualitatively similar trends between the longitudinal and circumferential directions. The tissues are on average more compliant along the longitudinal than the circumferential direction in the toe region. In the linear elastic phase, however, the tissues are stiffer along the longitudinal than the circumferential direction. The RV tissues do not show much difference in stiffness in the linear elastic phase. The toe region is much more elongated along the longitudinal than the circumferential direction in the RV tissue. 

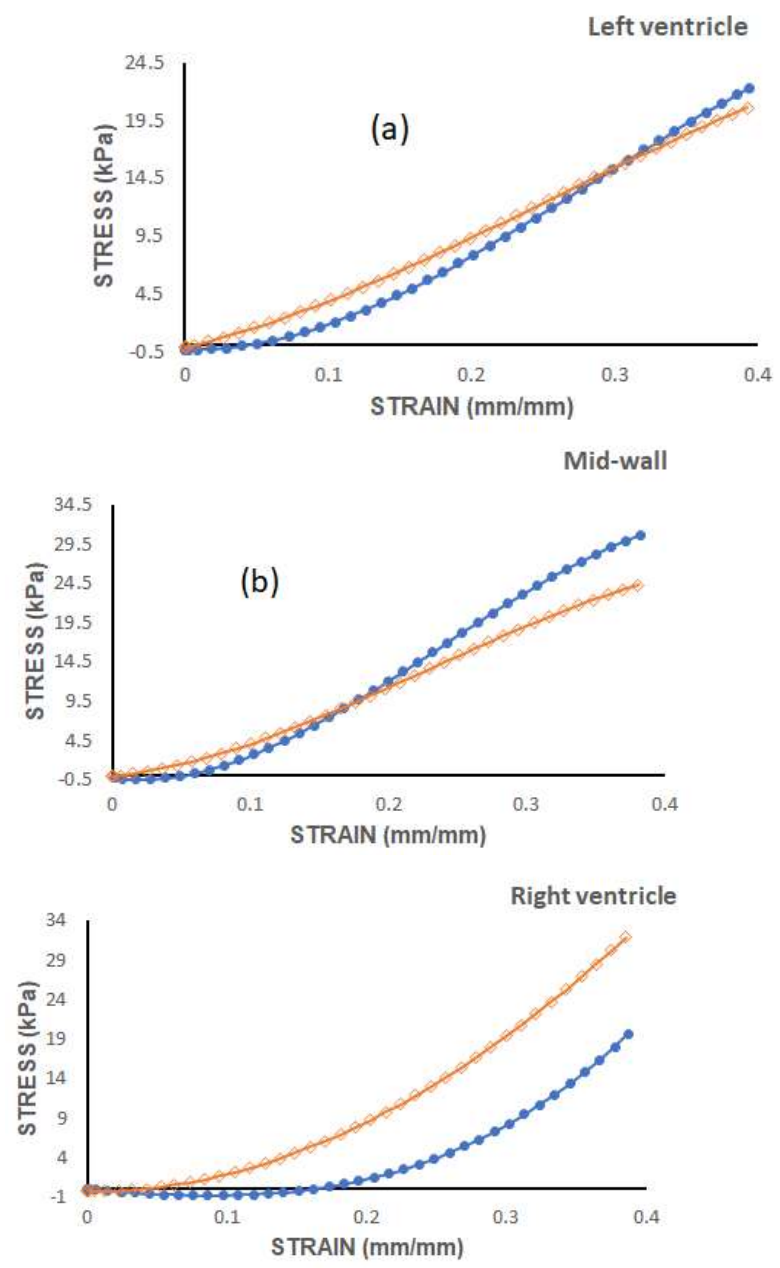

Figure 7. Longitudinal vs circumferential stress-strain curves for each heart wall's myocardium.

These differences are further qualitatively evaluated in this study by use of the singlefactor analysis of variance. Table 1 summarises the results in terms of the cross-directional variation of elastic modulus, peak stress at toe region limit and stress at $40 \%$ strain for each wall. The elastic modulus was determined from the linear elastic region for each heart wall specifically, from $12 \%$ to $37 \%$ strain for LV and MDW, and between $24 \%$ and $37 \%$ strain for RV. The toe region peak stresses were those corresponding to $12 \%$ strain for LV and MDW, those corresponding to $24 \%$ strain for RV. The stresses at $40 \%$ strain were obtained from the closest strain level. It is assumed that for a displacement-controlled test such as the one used in this study, it makes more sense to monitor the differences in the peak stresses than in the strains. The peak stresses are therefore used to reveal some nuances in the differences in the material behaviour during the straining process.

The results show that the MDW of rat myocardium exhibits statistically significant variation in elastic modulus between the longitudinal and circumferential directions. For LV and RV the variation is light but analyses of variance in the stresses yield significant variances in tissue responses at the toe region limit for the $\mathrm{LV}$, and both at the toe region limit and at $40 \%$ strain for the RV. 
Table 1. Statistical significance test results for axis-to-axis variations in biaxial mechanical parameters.

P-values: Cross-directional variation in a wall

\begin{tabular}{|c|c|c|c|}
\hline & LV & MDW & RV \\
\hline Elastic Modulus & 0.0601 & 0.0001 & 0.1128 \\
\hline $\begin{array}{l}\text { Peak stress at } \\
\text { toe region limit }\end{array}$ & 0.0042 & 0.2791 & 0.0000 \\
\hline $\begin{array}{l}\text { Peak stress at } \\
40 \% \text { strain }\end{array}$ & 0.9537 & 0.0342 & 0.0005 \\
\hline
\end{tabular}

Figure 8 shows indices of anisotropy which are calculated by relating tissue elastic moduli in the longitudinal and circumferential directions for each test. The results show that the MDW has the highest anisotropy averaged at around an index of 1.5. The LV and RV have average anisotropy indices of 1.3 and 0.9 , respectively.

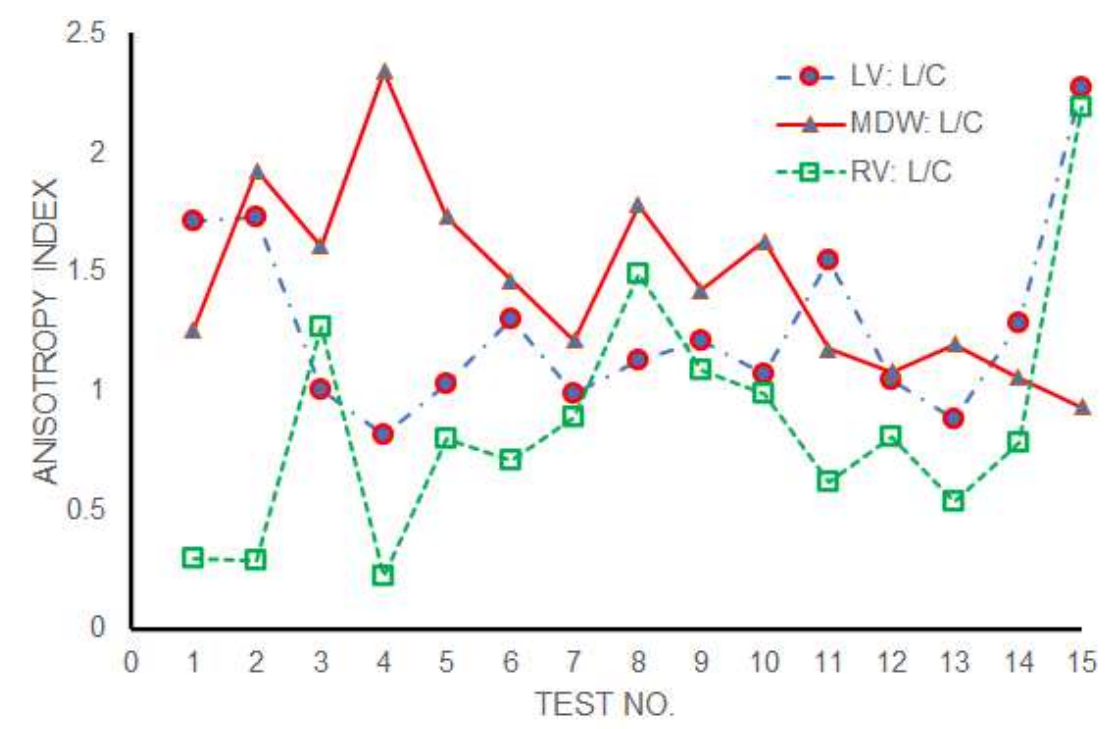

Figure 8. Anisotropy indices calculated over all tests for each heart wall.

\subsection{Cross-wall variation}

The tissue behaviour in the linear region is different for all the three wall tissues. The elastic moduli for the three walls in the longitudinal and circumferential directions are $69 \mathrm{kPa}$ and $58 \mathrm{kPa}$, respectively for $\mathrm{LV}, 109 \mathrm{kPa}$ and $77 \mathrm{kPa}$, respectively for MDW, and $103 \mathrm{kPa}$ and $129 \mathrm{kPa}$, respectively for RV. In terms of stress values at $40 \%$ strain the three tissue walls exhibit the following average values: $25 \pm 7.5 \mathrm{kPa}$ in longitudinal direction and $20 \pm 5 \mathrm{kPa}$ in circumferential direction for $\mathrm{LV} ; 32 \pm 7.5 \mathrm{kPa}$ in longitudinal direction and $25 \pm 5 \mathrm{kPa}$ in circumferential direction for $\mathrm{MDW}$; and $20 \pm 7 \mathrm{kPa}$ in longitudinal direction and $32 \pm 10 \mathrm{kPa}$ in circumferential direction for RV. These results show that the RV and MDW myocardial tissues in this study are stiffer than LV myocardial walls in both directions. The RV myocardial tissue is stiffest in the linear region in the circumferential direction. However, it is important to note that this is not the case in the toe region where the LV myocardial tissues are on average the stiffest in both directions. 
Table 2 summarises the significance tests for cross-wall variations of elastic moduli, toe region peak stresses, and stress at $40 \%$ strain for each direction. For elastic moduli, the results show that the only non-significant differences occur between the MDW and RV along the longitudinal direction. All the other cross-wall relationships yield significant differences for the elastic moduli. The peak stress at the toe region does not yield any significant difference between the LV and RV along the longitudinal direction. The results in the table show that from one wall to another, the most significant differences occur along the circumferential direction. This shows that each wall of the rat heart has distinct mechanical properties since there is at least one parameter that is different from wall to wall.

Table 2. Statistical significance test results for cross-wall variations in biaxial mechanical parameters.

\begin{tabular}{|c|c|c|c|c|c|c|}
\hline & \multicolumn{6}{|c|}{ P-values : Cross-wall variation in a particular direction } \\
\hline & \multicolumn{2}{|c|}{ LV-MDW } & \multicolumn{2}{|c|}{ LV-RV } & \multicolumn{2}{|c|}{ MDW-RV } \\
\hline & $\mathrm{L}$ & $\mathrm{C}$ & $\mathrm{L}$ & $\mathrm{C}$ & $\mathrm{L}$ & $\mathrm{C}$ \\
\hline $\begin{array}{l}\text { Elastic Mod- } \\
\text { ulus }\end{array}$ & 0.0000 & 0.0043 & 0.0142 & 0.0000 & 0.6552 & 0.0001 \\
\hline $\begin{array}{l}\text { Peak stress } \\
\text { at toe region }\end{array}$ & 0.0007 & 0.0111 & 0.8879 & 0.0001 & 0.0084 & 0.0033 \\
\hline limit & & & & & & \\
\hline $\begin{array}{l}\text { Peak stress } \\
\text { at } 40 \% \text { strain }\end{array}$ & 0.0021 & 0.2117 & 0.2119 & 0.0000 & 0.2864 & 0.0000 \\
\hline
\end{tabular}

$L$ and $C$, Longitudinal and circumferential

Ratios of cross-wall elastic moduli for all tests are shown in Figure 9. The graphs clearly show that the heart walls are distinct from each other in either direction. There is no particular ratio that lies close to unity for all cross-wall relationships shown in the figure. 

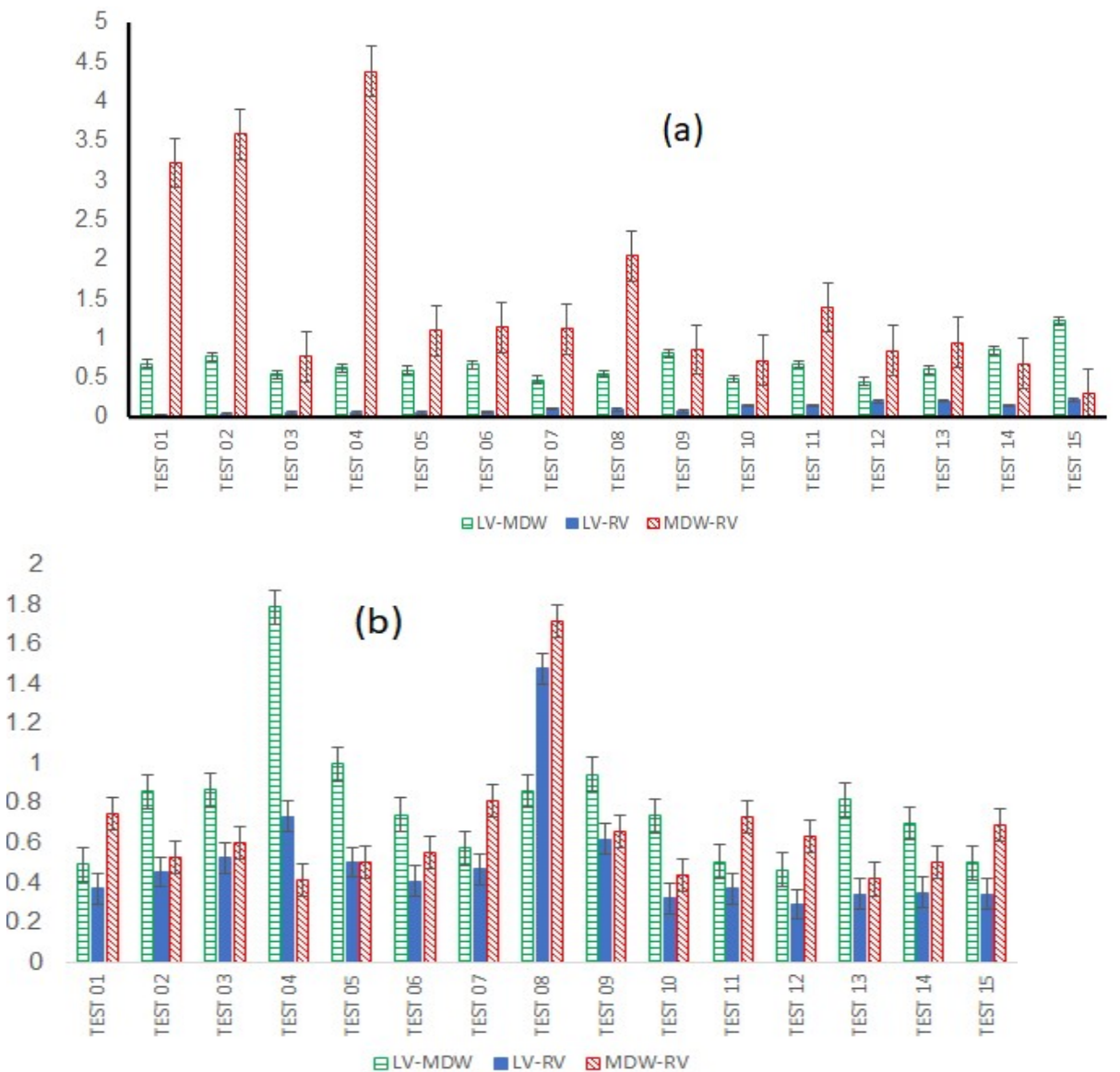

Figure 9. Cross-wall (a) longitudinal-to-longitudinal, and (b) circumferential-to-circumferential ratios between different heart walls for each test.

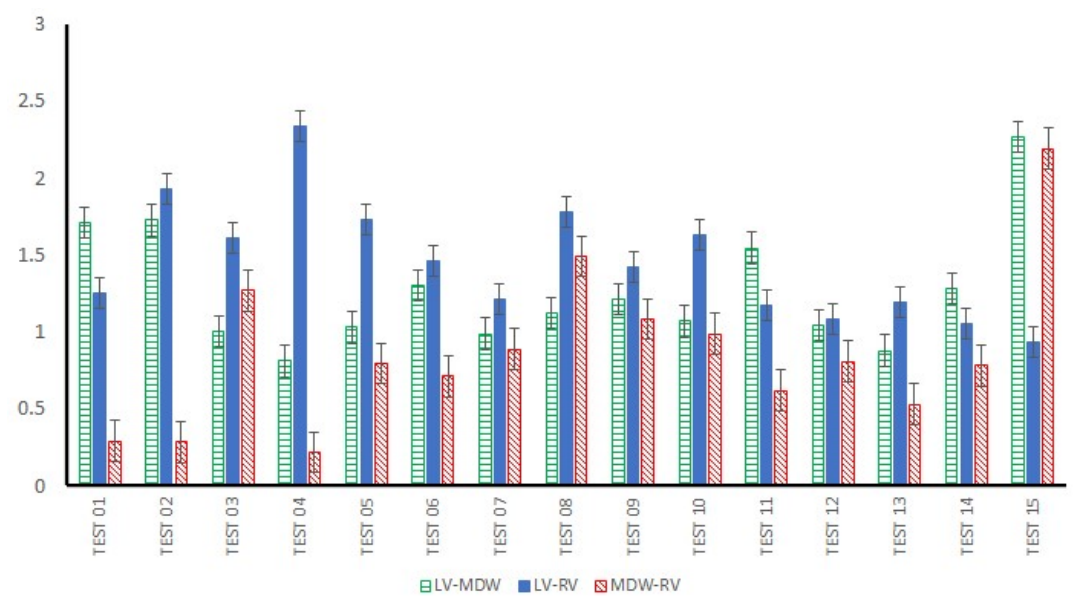

Figure 10. Index of anisotropy for all tests.

\subsection{Stored strain energy}

The passive response of the tissues can be linked to the amount of strain energy that can potentially be stored under load. In this study the stored strain energy in the toe and linear regions along both directions were evaluated and are tabulated in Table 3. The stored strain energy is numerically calculated using the trapezoidal rule given by,

$$
U_{T}=\sum_{n=1}^{N-1} \frac{1}{2}\left(\sigma_{i j}^{n+1}+\sigma_{i j}^{n}\right) \times\left(\varepsilon_{i j}^{n+1}-\varepsilon_{i j}^{n}\right)
$$


Where $\mathrm{n}$ is a sample point and $\mathrm{N}$ is the total length of the measured data.

The results in Table 3 show that the MDW stores the largest amount of strain energy along both directions. Despite its higher stiffness and relatively long toe region, the RV myocardium does have the least amount of stored strain energy except along the circumferential direction in the toe region. The LV stores much higher strain energy than the RV in the linear region, which is beneficial when one considers its relatively higher pumping demands.

Table 3. Stored strain energy values calculated within the toe region and linear elastic region for each heart wall myocardium.

\begin{tabular}{|c|c|c|c|c|}
\hline \multirow{2}{*}{$\begin{array}{l}\text { Wall of myocar- } \\
\text { dium }\end{array}$} & \multicolumn{2}{|c|}{ Toe region $(\mathrm{kJ})$} & \multicolumn{2}{|c|}{ Linear elastic region $(\mathrm{kJ})$} \\
\hline & Longitudinal & Circumferential & Longitudinal & Circumferential \\
\hline Left ventricle & 0.0803 & 0.2210 & 3.3130 & 3.4471 \\
\hline Mid-wall & 0.1941 & 0.3359 & 4.5341 & 3.7879 \\
\hline Right ventricle & 0.0728 & 0.7697 & 1.3251 & 2.8569 \\
\hline
\end{tabular}

\section{Discussion}

The results show that the stress-strain curves divert much further away from the average curve for both longitudinal and circumferential directions in all walls of the rat heart. This trend is typical for many soft tissue test results. The plotted SEM values demonstrate this diversion of the results with increased strain in the linear elastic phase. This is a cause for the difficulty with which myocardium tissue (or any soft tissue) behaviour can be estimated by any analytical model. Another dimension to this problem is the difficulties in standardising stress-strain curves of the myocardial tissue across two different laboratory testing conditions. It is therefore almost impossible to apply model parameters obtained from tests conducted in one laboratory to those obtained in a different laboratory. Even for tests obtained within the same laboratory, it is only reasonable to correlate different tests by examining how well those different tests fall within acceptable error bounds as demonstrated in this study, possibly within a region bounded by onestandard deviation error. A similar argument may be pertinent in the application of analytical or computational models for prediction of stress-strain behaviour of myocardium tissue.

The results show that cross-directional variances in the elastic modulus are statistically significant in the MDW, lightly significant in LV and non-significant in the RV. Since the tested specimens were dissected from the middle section of each wall (about the sagittal plane), it might be interesting to investigate the nature of the variances in different regions of each wall especially towards the MDW. Studies on other soft tissues such as valves have shown that the anisotropy varies over different regions [28]. However, the $\mathrm{p}$ value of 0.06 for the LV wall in this study may be interpreted differently with slight adjustment to the confidence region. In terms of cross-wall variation in the elastic modulus, only the MDW-RV does not yields any statistically different results along the longitudinal direction. 
The peak stresses in the toe region are extremely important since it is known that soft tissues typically operate within this region under physiological conditions. For cross-direction variation, it is observed that both the LV and RV yield statistically significant variances. These differences in the toe region behaviour within the LV and RV myocardium may have some significant influences in the contribution of the passive properties of the heart muscle towards the heart's pumping process. Cross-wall variation shows that only the longitudinal peak stresses between the LV and RV walls are statistically non-significant.

The behaviour of the myocardium tissue in the toe region along the longitudinal direction allows the heart to build up its volume without a corresponding increase in stress in the walls during diastole. The tissue in the circumferential direction develops a slightly higher strain to provide the necessary restitution force for pumping out of the blood during systole. The results in this study, show that the behaviour of the tissue walls in the linear region makes the heart stiffer and hard to stretch in the longitudinal direction. On the other hand, the heart tissue is relatively more compliant in the circumferential direction and more so up to $20 \%$ strain. These differences are caused by the different stiffness configurations in the two directions as statistically established in this study.

This study would have benefitted a great deal from histology studies of the rat myocardium at different times during the testing period to observe if there were other processes after tissue dissection that influenced its tensile behaviour. For example, some studies have reported the formation of fibrosis in the infarcted myocardium that eventually makes it stiff $[38,39]$.

The tested specimens were dissected roughly from the middle section of each wall, it might be interesting to investigate the nature of the variance in different regions of each wall especially towards the MDW where the elasticity cross-direction variation is statistically significant.

The tissues were tested in a bath that was maintained at $37^{\circ} \mathrm{C}$ to emulate the physiological conditions. However, it would be interesting to investigate the influence of different testing temperatures. Some of the test-related factors that were not considered in this investigation include the effect of the test specimen dimensions, clamping, different buffer solutions and stress relaxation.

\section{Conclusions}

The main aim of the current study was to investigate cross-directional and cross-wall variations in infarcted rat myocardium's passive tensile behaviour. These variations were investigated by examining any differences in the following parameters: anisotropy index, elastic moduli (stiffness), peak stress in the toe region, stress at $40 \%$ strain, and stored strain energy in the toe and linear regions. Anisotropy indices were only used for investigating cross-directional variations, as it would not make much practical sense to apply them across different directions. This study has three important findings: (1) LV and MDW tissues have relatively shorter toe regions of $10-15 \%$ strain as compared to RV tissue whose toe region extends up to twice as much as that (2) LV tissues have higher strain energy storage in the linear region despite being lower in stiffness than the RV (3) the MDW has the highest strain energy storage along both directions which might be directly related to its high level of anisotropy.

This paper shows that the myocardium tissue has both cross-directional and crosswall variations. This may explain why analytical models that implement anisotropy tend to yield much better fit than their counterparts that assume isotropy. Thus, the application of models that implement both dependencies are recommended for the modelling of myocardium tissue. An equally important finding is that the passive behaviour of myocardium tissue under tensile testing may be hard to reproduce across different laboratory conditions. Even in a laboratory, it might be prudent to consider validating repeatability 
of test results by examining how well they lie within one-standard deviation of the average stress-strain curve.

Supplementary Materials: No supplementary data is available except data that is available here https://doi.org/10.17632/y753k82jt4.1

Author Contributions: Conceptualization, F.N; T.F; and N.D;. methodology, F.N and H.N.; software H.N; T.P; and F.N; validation, F.N; I.M; T.P; and H.N.; formal analysis, TP; F.N; and H.N.; investigation, F.N and H.N.; resources, N.D; and F.N; data curation, F.N; H.N; I.M and M.M;.; writing-original draft preparation, F.N and H.N.; writing-review and editing, M.M; I.M; T.P; and F.N;.; visualization, F.N; H.N and T.P;.; supervision, F.N; N.D; and T.F; and H.N.; project administration, F.N; and H.N,.; funding acquisition, N.D; and F.N;. All authors have read and agreed to the published version of the manuscript.

Funding: This research was funded by National Research Foundation (NRF), grant number 129380

Institutional Review Board Statement: The study was conducted according to the guidelines of the Declaration of Helsinki, and this study was approved by the Faculty of Health Sciences Animal Ethics Committee of the University of Cape Town on 6 May 2019 under reference number FHS AEC REF 019-019.

Informed Consent Statement: Not applicable.

Data Availability Statement: No supplementary data is available except data that is available here https://doi.org/10.17632/y753k82jt4.1

Acknowledgments: Support from the National Research Foundation (NRF) Grant number (129380) is gratefully acknowledged. Unisa CAPEX Programme supported the acquisition of biaxial testing machine in the Department of Mechanical Engineering, School of Engineering, College of Science Engineering and Technology.

Conflicts of Interest: The authors declare no conflict of interest.

\section{References}

1. Nemavhola, F., Mechanics of the septal wall may be affected by the presence of fibrotic infarct in the free wall at end-systole. International Journal of Medical Engineering and Informatics, 2019. 11(3): p. 205-225.

2. Nemavhola, F., Fibrotic infarction on the LV free wall may alter the mechanics of healthy septal wall during passive filling. Biomedical materials and engineering, 2017. 28(6): p. 579-599.

3. Nemavhola, F., Biaxial quantification of passive porcine myocardium elastic properties by region. Engineering Solid Mechanics, 2017. 5(3): p. 155-166.

4. Ndlovu, Z., F. Nemavhola, and D. Desai, BIAXIAL MECHANICAL CHARACTERIZATION AND CONSTITUTIVE MODELLING OF SHEEP SCLERA SOFT TISSUE. Russian Journal of Biomechanics/Rossijski Zurnal Biomehaniki, 2020. 24(1).

5. Ngwangwa, H.M. and F. Nemavhola, Evaluating computational performances of hyperelastic models on supraspinatus tendon uniaxial tensile test data. Journal of Computational Applied Mechanics, 2021. 52(1): p. 27-43.

6. Nemavhola, F., H.M. Ngwangwa, and T. Pandelani, An Investigation of Uniaxial Mechanical Properties of Excised Sheep Heart Muscle Fibre-Fitting of Different Hyperelastic Constitutive Models. Preprints, 2021. 2021080566 (doi: 10.20944/preprints202108.0566.v1).

7. Nemavhola, F. and R. Sigwadi, Prediction of hyperelastic material properties of Nafion117 and Nafion/ZrO2 nano-composite membrane. International Journal of Automotive and Mechanical Engineering, 2019. 16(2): p. 6524-6540.

8. Wang, H., et al., Bioengineered analog of stromal cell-derived factor $1 \alpha$ preserves the biaxial mechanical properties of native myocardium after infarction. Journal of the Mechanical Behavior of Biomedical Materials, 2019. 96(February): p. 165-171. 
9. Martynenko, A. and V.V. Zozulya, Mathematical modeling of the cardiac tissue. Mechanics of Advanced Materials and Structures, 2021: p. 1-17.

10. Childers, R.C., et al., Paired pressure-volume loop analysis and biaxial mechanical testing characterize differences in left ventricular tissue stiffness of volume overload and angiotensin-induced pressure overload hearts. Journal of Biomechanical Engineering, 2021. 143(8): p. 1-10.

11. Jorba, I., et al., In Vitro Methods to Model Cardiac Mechanobiology in Health and Disease. Tissue Engineering - Part C: Methods, 2021. 27(3): p. 139-151.

12. Li, W., Biomechanics of infarcted left Ventricle-A review of experiments. Journal of the Mechanical Behavior of Biomedical Materials, 2020. 103(October 2019): p. 103591.

13. Nemavhola, F., Pig sclera stress-strain dataset under biaxial tensile testing. Mendeley Data, 2020. V1.

14. Masithulela, F.J., Computational biomechanics in the remodelling rat heart post myocardial infarction. 2016.

15. Masithulela, F., Bi-ventricular finite element model of right ventricle overload in the healthy rat heart. Bio-medical materials and engineering, 2016. 27(5): p. 507-525.

16. Masithulela, F. The effect of over-loaded right ventricle during passive filling in rat heart: A biventricular finite element model. in ASME International Mechanical Engineering Congress and Exposition. 2015. American Society of Mechanical Engineers.

17. Masithulela, F. Analysis of passive filling with fibrotic myocardial infarction. in ASME international mechanical engineering congress and exposition. 2015. American Society of Mechanical Engineers.

18. Masithulela, F. The Effect of Over-Loaded Right Ventricle During Passive Filling in Rat Heart: A Biventricular Finite Element Model. in ASME 2015 International Mechanical Engineering Congress and Exposition. 2015.

19. Nemavhola, F., Detailed structural assessment of healthy interventricular septum in the presence of remodeling infarct in the free wall-A finite element model. Heliyon, 2019. 5(6): p. e01841.

20. Nemavhola, F., et al., Passive Biaxial Tensile Dataset of Three Main Rat Heart Myocardia: Left Ventricle, Mid-Wall and Right Ventricle. Preprints, 2021. 2021080153(Version 1).

21. Rigolin, V.H., et al., The forgotten chamber: The importance of the right ventricle. Catheterization and Cardiovascular Diagnosis, 1995. 35(1): p. 18-28.

22. Sheehan, F. and A. Redington, The right ventricle: Anatomy, physiology and clinical imaging. Heart, 2008. 94(11): p. $1510-1515$.

23. Mas, P.T., J.F. Rodríguez-Palomares, and M.J. Antunes, Secondary tricuspid valve regurgitation: A forgotten entity. Heart, 2015. 101(22): p. 1840-1848.

24. Kakaletsis, S., et al., Right ventricular myocardial mechanics: Multi-modal deformation, microstructure, modeling, and comparison to the left ventricle. Acta Biomaterialia, 2021. 123: p. 154-166.

25. Sacks, M.S. and C.J. Chuong, Biaxial Mechanical Properties of Passive Right Ventricular Free Wall Myocardium. Journal of Biomechanical Engineering, 1993. 115(May 1993): p. 202-204.

26. Sacks, M.S. and C.J. Chuong, A constitutive relation for passive right-ventricular free wall myocardium. Journal of Biomechanics, 1993. 26(11): p. 1341-1345.

27. Golob, M., R. Moss, and N. Chelser, Materials Science Perspective. Annals of Biomedical Engineering, 2010. 42(10): p. 29863005.

28. Laurence, D., et al., An investigation of regional variations in the biaxial mechanical properties and stress relaxation behaviors of porcine atrioventricular heart valve leaflets. Journal of Biomechanics, 2019. 83: p. 16-27.

29. Nemavhola, F., Study of biaxial mechanical properties of the passive pig heart: material characterisation and categorisation of regional differences. International Journal of Mechanical and Materials Engineering, 2021. 16(1).

30. Xie, T. and H. Zaidi, Age-dependent small-animal internal radiation dosimetry. Molecular Imaging, 2013. 12(6): p. $364-375$.

31. Goverment, S.A., Animals Protection Act Act 71 (1962). Goverrment Gazette, 1962. 71: p. 1 -13. 
32. Government, S.A. Animal protection index (API) 2020. 2020.

33. Gardiner, J.C. and J.A. Weiss, Simple shear testing of parallel-fibered planar soft tissues. Journal of Biomechanical Engineering, 2001. 123(2): p. 170-175.

34. Viiidik, A., Biomechanics of tendons and other soft connective tissues. Testing methods and structure-function interdependence., in Biomechanics: Basic and Applied Research, G. Bergmann, R. Kolbel, and A. Rohlmann, Editors. 1987, Kluwer Academic Publishers Group: Dordrecht. p. 59-72.

35. Fung, Y.C., A first course in continuum mechanics for physical and biological engineers and scientists. 1994, Englewood Cliffs, N.J.: Prentice Hall.

36. Montgomery, D.C. and G.C. Runger, Applied statistics and probability for engineers (with CD). 2007: John Wiley \& Sons.

37. Jiang, M., et al., Clamping soft biologic tissues for uniaxial tensile testing: A brief survey of current methods and development of a novel clamping mechanism. Journal of the Mechanical Behavior of Biomedical Materials, 2020. 103: p. 103503.

38. Sirry, M.S., et al., Characterisation of the mechanical properties of infarcted myocardium in the rat under biaxial tension and uniaxial compression. Journal of the Mechanical Behavior of Biomedical Materials, 2016. 63: p. 252-264.

39. Martonová, D., et al., Passive mechanical properties in healthy and infarcted rat left ventricle characterised via a mixture model. Journal of the Mechanical Behavior of Biomedical Materials, 2021. 119: p. 104430. 